\title{
Comparación entre el contenido ruminal y fecal en la composición botánica de la dieta de un huemul
}

\author{
Comparison of ruminal and fecal content in the \\ botanical composition of the diet of one huemul
}

Laura Borrelli ${ }^{1}$, Leandro Lema ${ }^{2}$, Mauricio Berardi ${ }^{2}$, Iván Hoermann², Juan Salinas² \& Alejandro Vila ${ }^{3}$

Hippocamelus bisulcus (Molina, 1782) es un ciervo endémico de Chile y Argentina que se distribuía originalmente en la región de los bosques andino patagónicos y las áreas ecototonales con la estepa (Cabrera \& Yepes, 1960). Hacia comienzos del siglo XX su distribución se redujo significativamente debido a presiones antrópicas (Díaz, 2000). En la actualidad, las poblaciones remanentes son pequeñas y están fragmentadas (Vila et al. 2006). Por esta razón, el huemul ha sido clasificado como "en peligro" a nivel nacional e internacional (Glade, 1988; Díaz \& Ojeda, 2000; IUCN, 2016).

La mayoría de los estudios sobre la composición dietaria del huemul han sido realizados mediante el análisis microhistológico de heces (Colomes, 1978; Merino, 1995; López et al. 2001; Smith-Flueck, 2003; Galende et al. 2005; Vila et al. 2009a, 2009b). Otros autores han empleado técnicas de observación directa de animales durante periodos de alimentación (Frid, 1994; Van Winden, 2006). Si bien el análisis del contenido ruminal es una opción que brinda mayor exactitud para la determinación de la dieta de un herbívoro silvestre, este implica el sacrificio de animales (Holechek et al. 1982). Consecuentemente, debido al estatus de conservación de la especie, esta técnica no puede ser utilizada para evaluar la dieta del huemul. En esta comunicación describimos la composición botánica del contenido estomacal e intestinal de un macho adulto de huemul que fue hallado muerto en el Parque Nacional Los Alerces, Argentina.

Este parque nacional está dominado por cordones montañosos y lagos de origen glaciario.
La temperatura media anual es de $8^{\circ} \mathrm{C}$ y las precipitaciones varian entre 3.000 y $800 \mathrm{~mm}$ anuales en un gradiente oeste-este. El área de estudio está dominada por bosques puros o mixtos de ciprés de la cordillera (Austrocedrus chilensis) y coihue (Nothofagus dombeyi). El huemul presenta una amplia distribución dentro de la misma pero se desconoce el tamaño de sus poblaciones (Pastore et al. 2005).

El ejemplar estudiado fue encontrado muerto el 8 de enero del 2009 sobre la costa del Lago Futalaufquen $\left(42^{\circ} 49^{\prime} 21^{\prime}\right.$ 'S, 71 $37^{\prime}$ $\left.53^{\prime \prime} \mathrm{O}\right)$. Tanto su estado de conservación como nutricional eran muy buenos, su peso fue de 68 kg y la edad estimada de 5-6 años (Berardi \& Hoermann, 2009). Se le practicó una necropsia pero no pudieron determinarse las causas de su muerte. Se colectó una muestra compuesta de dos sectores del rumen y otra de heces del intestino y el recto. Las muestras del contenido estomacal fueron lavadas con agua caliente para eliminar enzimas digestivas que dificultan la confección de los preparados microscópicos

Instituto Nacional de Tecnología Agropecuaria (INTA), EEA-Bariloche, Modesta Victoria 4450, (8400) Bariloche, Río Negro, Argentina. lborrelli@bariloche.inta.gov.ar

2 Administración de Parques Nacionales (APN), Parque Nacional Los Alerces, Chubut, Argentina. mauricioberardi@gmail.com

3 Wildlife Conservation Society, Balmaceda 586, Punta Arenas, Chile. $\sum$ avila@wcs.org 
(Pelliza Sbriller, 1993). El procesamiento de las mismas se realizó según Sparks \& Malechek (1968), Williams (1969) y Latour y Pelliza Sbriller (1981). La composición botánica de la dieta fue obtenida por medio del análisis microhistológico. Se montaron cinco preparados por muestra y se observaron 20 campos microscópicos por cada uno de ellos, a 100 aumentos (Holechek \& Vavra, 1981). Se identificaron fragmentos epidérmicos y no epidérmicos de las plantas ingeridas (Sparks \& Malechek, 1968; Sepúlveda et al. 2004) y se cuantificó su frecuencia relativa (Holechek \& Gross, 1982).

Se registró el consumo de 19 y 17 especies vegetales en las muestras de estómago y heces respectivamente (Tabla 1). En ambas muestras, el mayor número de especies se observó para las clases forrajeras leñosas (55 al $56 \%$ de los fragmentos en las muestras) y herbáceas (43 al $44 \%$ ). Erodium cicutarium y Medicago sp. sólo fueron registradas en las heces. En cambio, Fucsia magellanica, Erygeron sp., Gunnera magellanica y una gramínea no identificada sólo se observaron en el rumen. El huemul bajo estudio consumió predominantemente Nothofagus sp., Gaultherya sp., Maytenus sp., Ribes magellanica, Acaena sp. y Anemone sp. (Tabla 1). El consumo de gramíneas fue menor al $1 \%$ y no se registró la presencia de graminoideas. Algunas especies fueron más abundantes en las heces que en el rumen y viceversa (Tabla 1). Las diferencias más marcadas se observaron en Discaria sp., Maytenus sp., Nothofagus sp., R. magellanica, Acaena sp., Anemone sp. y una hierba no identificada.

La dieta obtenida en este estudio no se diferencia de la reportada para otras poblaciones (Vila et al. 2009a, 2010). Sin embargo, el contenido de hierbas fue mayor que el observado previamente para esta misma área de estudio $(2,7 \%$ en Vila et al. 2009a). Las diferencias observadas con los datos reportados para esta misma área podrían estar ocasionadas por el bajo número de muestras disponibles en este estudio (un solo ejemplar).

También es posible que algunas de las especies identificadas en el rumen no sean encontradas en las heces por efecto de la digestión (Mc Innis et al. 1983). Sin embargo, no es esperable que las especies identificadas en las heces no se registren en el rumen. La aparición de Erodium sp. y Medicago sp. sólo en heces podría explicarse por los errores asociados al método, ya que en ambos casos sus frecuencias de aparición son extremadamente bajas. Estas diferencias también se podrían adjudicar al diferimiento temporal, y potencialmente espacial, del forraje consumido y encontrado en el estómago y las heces.

$\mathrm{Si}$ bien se desconocen los tiempos de retención del alimento para el huemul, aquellos reportados para otros ungulados comprenden un rango de entre 19 y 105 horas (Huston et al. 1986; Wenninger \& Shipley, 2000). Considerando

Tabla 1. Composición porcentual de la dieta de un macho de huemul del Parque Nacional Los Alerces.

\begin{tabular}{lcc}
\hline & Estómago & Heces \\
\hline GRAMINEAS & $\mathbf{0 , 5 1}$ & $\mathbf{0 , 8 3}$ \\
Gramínea s/identificar & 0,38 & 0,00 \\
Poa sp. & 0,13 & 0,83 \\
ARBOLES Y ARBUSTOS & $\mathbf{5 5 , 3 2}$ & $\mathbf{5 6 , 0 8}$ \\
Discaria sp. & 5,19 & 0,55 \\
Fucsia magellanica & 0,13 & 0,00 \\
Gaultherya sp. & 9,62 & 7,73 \\
Maytenus sp. & 20,13 & 14,92 \\
Nothofagus sp. & 15,06 & 19,89 \\
Ribes magellanica & 4,43 & 10,77 \\
Senecio sp. & 0,76 & 2,21 \\
HIERBAS & $\mathbf{4 4 , 1 8}$ & $\mathbf{4 3 , 0 9}$ \\
Acaena sp. & 5,06 & 11,33 \\
Anemone sp. & 19,11 & 12,15 \\
Chlorea sp. & 3,42 & 0,55 \\
Erodium cicutarium & 0,00 & 1,10 \\
Erygeron sp. & 0,13 & 0,00 \\
Fruto s/identificar & 2,15 & 1,93 \\
Gunnera magellanica & 1,39 & 0,00 \\
Hierba s/identificar 1 & 2,78 & 1,93 \\
Hierba s/identificar 2 & 8,86 & 4,70 \\
Medicago sp. & 0,00 & 0,83 \\
Osmorhyza sp. & 0,13 & 5,52 \\
Vicia nigricans & 1,14 & 3,04 \\
\hline TOTAL & $\mathbf{1 0 0}$ & $\mathbf{1 0 0}$ \\
\hline & & \\
\hline
\end{tabular}


la relación alométrica existente entre el tiempo de retención de alimento y el tamaño corporal de rumiantes africanos (Gordon \& Illius, 1994), el tiempo de retención de un huemul macho de 75 $\mathrm{kg}$ podría estimarse en unas 40 horas. Esto sugiere que habría un tiempo suficiente entre el consumo de un tipo de vegetación y/o el consumo en un sitio particular como para encontrarse diferencias entre la composición de la dieta en el estómago y en las heces.

Este trabajo constituye el primer antecedente en el que se compara la composición botánica de la dieta obtenida a partir del rumen y las heces de un huemul. Dado que se dispone de una única muestra, son evidentes las limitaciones del estudio. $\mathrm{Si}$ bien resulta necesario analizar más ejemplares para confirmar estos resultados, considerando que se trata de una especie en peligro de extinción y la obtención de más muestras depende del encuentro fortuito y casual de animales recientemente muertos, existen pocas posibilidades de que esto ocurra. Considerando estas limitaciones, se sugiere que el análisis de heces brinda información fidedigna de la dieta a nivel de grupos funcionales (gramíneas, árboles y arbustos, hierbas), donde las diferencias con el contenido ruminal fueron mínimas. Sin embargo, debe señalarse que, a nivel de especies, se presentan algunas diferencias.

\section{AGRADECIMIENTOS}

Agradecemos a Eduardo Ramilo (APN), Hernán Pastore (APN) y Elizabeth Chang Reissig (CONICET) por trasladar y conservar las muestras, y a Donaldo Bran (INTA) por colaborar en la identificación de las plantas del rumen; como así también las sugerencias de dos revisores anónimos para mejorar este trabajo.

\section{LITERATURA CITADA}

Berardi, M., \& Hoermann, I. (2009). Informe necropsia de huemul (Hippocamelus bisulcus). Villa Futalaufquen, Argentina: Programa Conservación del Huemul, Parque Nacional Los Alerces, APN.

Cabrera, A., \& Yepes, J. (1960). Mamíferos Sudamericanos ( $2^{\mathrm{a}}$ end). Buenos Aires, Argentina: Ediar.
Colomes, A. (1978). Biología y ecología del huemul chileno (Hippocamelus busulcus): Estudios de sus hábitos alimentarios. Tesis de doctorado. Santiago, Chile: Universidad de Chile.

Díaz, N. I. (2000). El huemul (Hippocamelus bisulcus Molina, 1782): Una perspectiva histórica. En N. I. Díaz \& J. A. Smith-Flueck (Eds.), El huemul Patagónico, un misterioso cérvido al borde de la extinción (pp. 1-32). Buenos Aires, Argentina: Literature of Latin America.

Díaz, G. B., \& Ojeda, R. A. (2000). Libro Rojo: Mamíferos amenazados de la Argentina. Mendoza, Argentina: SAREM.

Galende, G., Ramilo, E., \& Beati, A. (2005). Diet of Huemul Deer (Hippocamelus bisulcus) in Nahuel Huapi National Park, Argentina. Studies on Neotropical Fauna and Environment, 40, 1-5.

Glade, A. (1988). Libro Rojo de los Vertebrados Terrestres de Chile. Santiago, Chile: Corporación Nacional Forestal.

Frid, A. (1994). Observations on habitat use and social organization of a huemul (Hippocamelus bisulcus) coastal population in Chile. Biological Conservation, 67, 1319.

Gordon, I. J., \& Illius, A. W. (1994). The functional significance of the browser-grazer dichotomy in African ruminants. Oecologia, 98, 167175.

Holechek, J. L., \& Vavra, M. (1981). The effect of slide and frequency observation numbers on the precision of microhistological analysis. Journal of Range Management, 34, 337338.

Holechek, J. L., \& Gross, V. (1982). Evaluation of different calculation procedures for microhistological analysis. Journal of Range Management, 35, 721-723.

Holechek, J. L., Vavra, M., \& Pieper, R. D. (1982). Botanical composition determination of range herbivore diets: A review. Journal of Range Management, 35, 309-315.

Huston, J. E., Rector, B. S., Ellis, W. C., \& Allen, M. L. (1986). Dynamics of digestion in cattle, sheep, goats and deer. Journal of Animal Science, 62, 208-215. 
IUCN (2016). The IUCN Red List of Threatened Species. Version 2016-1. IUCN. <www. iucnredlist.org $>$. Accessed 2 July 2016.

Latour, M. C., \& Pelliza Sbriller, A. (1981). Clave para la determinación de la dieta de herbivoros en el Noroeste de la Patagonia. Revista de Investigaciones Agropecuarias INTA, 1, 109-157.

López, R., Figueroa, R., Corales, E. S., Trostel, A., \& Alvarado, S. (2001). Estudio del huemul en los Nevados de Chillán - Laguna de la Laja. Concepción, Chile: CODEFF \& Sociedad Zoológica de Frankfurt.

Mc Innis, M. L., Vavra, M., \& Kueger, W. C. (1983). A comparison of four methods used to determine the diets of large herbivores. Journal of Range Management, 36, 302306.

Merino, M. (1995). Dieta del huemul(Hippocamelus bisulcus) en el Canal Moyano - Glaciar Viedma, Parque Nacional Los Glaciares, Provincia de Santa Cruz, Argentina. Boletín Técnico FVSA, 25, 18-25.

Pastore, H., Ramilo, E. J., \& Vila, A. R. (2005). Distribución actual del huemul en los Parques Nacionales de Argentina. Bariloche, Argentina: Administración de Parques Nacionales \& WCS.

Pelliza Sbriller, A. (1993). Acerca de la Microhistología. Bariloche, Argentina: INTA, Estación Experimental Agropecuaria Bariloche.

Sepúlveda, L., Pelliza, A., \& Manacorda, M. (2004). Importancia de los tejidos no epidérmicos en el microanálisis de dieta. Ecología Austral, 14, 31-38.

Smith-Flueck, J. A. (2003). La ecología del huemul (Hippocamelus bisulcus) en la Patagonia Andina de Argentina y consideraciones sobre su conservación. Tesis de doctorado. Bariloche, Argentina: Universidad Nacional del Comahue.

Sparks, D., \& Malechek, J. C. (1968). Estimating percentage dry weight in diets using a microscopic technique. Journal of Range Management, 21, 264-265.

Van Winden, J. (2006). Diet and habitat of the huemul (Hippocamelus bisulcus) in Bernardo O' Higgins National Park, Chile. Tesis de magíster. Utrecht, Holanda: Utrecht University.

Vila, A. R., López, R., Pastore, H., Faúndez, R., \& Serret, A. (2006). Current distribution and conservation of the huemul (Hippocamelus bisulcus) in Argentina and Chile. Mastozoología Neotropical, 13, 263-269.

Vila, A. R., Galende, G., \& Pastore, H. (2009a). Feeding ecology of the endangered huemul (Hippocamelus bisulcus) in Los Alerces National Park, Argentina. Mastozoología Neotropical, 16, 423-431.

Vila, A. R., Borrelli, L., \& Martínez, L. (2009b). Dietary overlap between huemul and livestock in Los Alerces National Park. Journal of Wildlife Management, 73, 368-373.

Vila, A. R., Saucedo Galvez, C. E., Aldridge, D., Ramilo, E., \& Corti González, P. (2010). South Andean Huemul (Hippocamelus bisulcus, Molina 1782). In J. M. Barbanti Duarte \& S. González (Eds.), Neotropical Cervidology: Biology and Medicine of Latin American Deer (pp. 89-100). San Pablo, Brasil: FUNEP-IUCN.

Wenninger, P. S., \& Shipley, L. A. (2000). Harvesting, rumination, digestion, and passage of fruit and leaf diets by a small ruminant, the blue duiker. Oecologia, 123, 466-474

Williams, O. B. (1969). An improved technique for identification of plant fragments in herbivore feces. Journal of Range Management, 22, 51-52. 